\title{
Elastic Spectrum Allocation for Variable Traffic in Flexible-Grid Optical Networks
}

\author{
L. Velasco ${ }^{1 *}$, M. Klinkowski ${ }^{2}$, M. Ruiz ${ }^{1}$, V. López ${ }^{3}$, G. Junyent ${ }^{1}$ \\ ${ }^{1}$ Universitat Politècnica de Catalunya (UPC), Barcelona, Spain \\ ${ }^{2}$ National Institute of Telecommunications, Warsaw, Poland \\ ${ }^{3}$ Telefónica I+D, Madrid, Spain \\ *e-mail:Ivelasco@ac.upc.edu
}

\begin{abstract}
We focus on lightpath adaptation under variable traffic demands in flexible-grid optical networks. Specifically, we study three different adaptation schemes for the variable spectrum allocation. Illustrative numerical results show the benefits of the schemes. (C) 2011 Optical Society of America

OCIS codes: (060.4510) Optical communications; (060.4251) Networks, assignment and routing algorithms
\end{abstract}

\section{Introduction}

The currently deployed wavelength division multiplexing (WDM) optical networks operate within a rigid frequency grid and with single-line-rate transponders making use of single carrier modulation techniques. The evolution path of optical transport networks can be translated to the application of advanced single-carrier modulation formats (such as QPSK, 16-QAM) in mixed-line-rate networks, the introduction of multi carrier modulation techniques (such as O-OFDM), and the elastic access to spectral resources within flexible frequency grids. Thanks to these advances, future elastic optical networks (EONs) will utilize the spectral resources efficiently, according to the transmission path characteristics and bandwidth requirements [1, 2]; EON divides the spectrum into frequency slots which provides greater granularity than rigid networks based on WDM.

In EON operating within a flexible grid, the optical path (lightpath) is determined by its routing path and the allocated fraction of frequency spectrum (i.e., the subset of frequency slots allocated) around a central frequency (CF). The introduction of EON opens new functionalities to be developed at the optical layer, such as the adaptation of lightpaths through appropriate spectrum allocation (SA) in a response to bandwidth variations, in particular, expansion/reduction of the spectrum when the required bit rate of a demand increases/decreases [3].

In this paper, we define three schemes for variable SA in EON, namely: 1) Fixed SA, 2) Semi-elastic SA, and 3) Elastic SA. We compare the performance of these schemes assuming a multi-hour traffic profile. The consideration of multiple time intervals results in the time-dependent routing with ELastIc Spectrum Allocation (ELISA) problem.

\section{The ELastIc Spectrum Allocation (ELISA) problem}

The ELISA problem can be formally stated as follows.

Given: 1) an EON represented by a graph $G(V, E)$, being $V$ the set of optical nodes and $E$ the set of fiber links connecting two nodes in $V ; 2$ ) an ordered set $S$ of frequency slots in each link in $E$; 3) a set of time intervals $T$; 4) a set $D$ of demands to be transported; each demand $d$ is represented by a tuple $\left(s_{d}, t_{d},\left\{h_{d t}: \forall t \in T\right\}, h_{d}{ }^{\text {min }}\right)$, where $s_{d}$ and $t_{d}$ are the source and the destination nodes respectively, $h_{d t}$ is the requested bandwidth in time interval $t\left(h_{d t} \leq h_{d}{ }^{\max }\right)$, and $h_{d}{ }^{\min }$ is the minimum bandwidth to be satisfied in every time interval, otherwise the demand will be rejected.

Find the route over the EON and the spectrum allocation of every transported demand in each interval time.

Objective: minimize the number of rejected demands (primary objective) and the amount of un-served bandwidth (secondary objective).

To solve the ELISA problem, we consider three schemes for variable in time SA (Fig. 1) that put some restrictions on the assigned CF and SA:

- Fixed SA (Fig. 1a) both the assigned CF and AS do not change in time; each slot is rigidly assigned to one demand at most and it is not shared between two demands. At each time period, demands can use all or only part of the allocated spectrum to convey the bandwidth requested for that period.

- Semi-elastic SA (Fig. 1b) the assigned CF is fixed but the spectrum width may vary; its total width is limited to a spectrum range. Here, half-slots are defined so increments/decrements of one slot are achieved by allocating/releasing one half-slot at each end of the already allocated spectrum while keeping invariant the

The research leading to these results has received funding from the European Community's Seventh Framework Programme FP7/2007-2013 under grant agreement $\mathrm{n}^{\circ} 247674$ STRONGEST project. Moreover, it was supported by the Spanish science ministry through the TEC2011-27310 ELASTIC project and by the Polish Ministry of Science and Higher Education. 


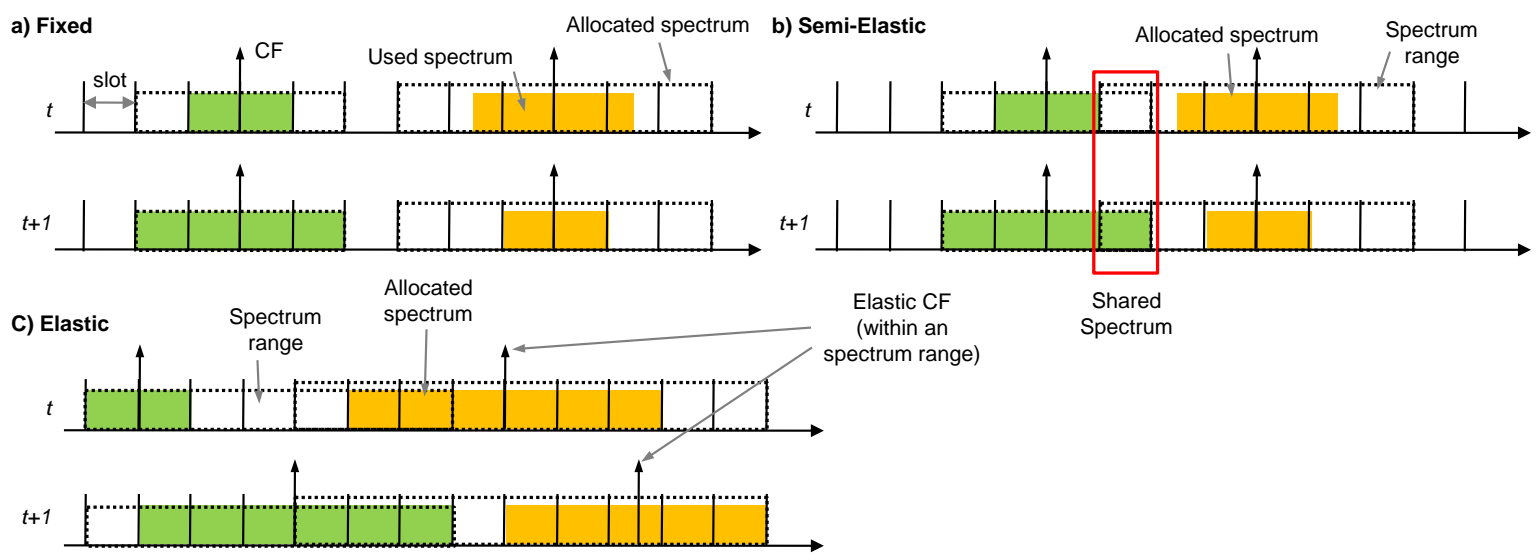

Fig. 1. Three schemes for variable spectrum allocation in a flexible-grid network (two time intervals are observed, $t$ and $t+1$ ).

CF. In this scheme, half-slots can be shared between two neighboring demands where its defined spectrum ranges overlap, but used by, at most, one demand in a time interval.

- Elastic SA (Fig. 1c) both the assigned CF and the allocated spectrum are flexibly selected at each time interval (the allocated spectrum can be flexibly moved within the spectrum range); a slot can be shared between two or more demands, but used by, at most, one demand in a time interval.

The problem concerns the resolution of the static Routing and Spectrum Allocation (RSA), based on [4], for each time period in which meaningful bandwidth variations are observed. Apart from the common RSA constraints, such as the spectrum continuity and contiguity, additional constraints related to the degree of flexibility in the spectrum allocation arise in the ELISA problem. Since Integer Linear Programming (ILP) formulations of ELISA are difficult to be solved, as an alternative we propose three heuristic algorithms based on the BRKGA meta-heuristic [5]. The performance of the proposed heuristics was validated against optimal solutions obtained with ILP models for small topologies. In all the tests performed, heuristic solutions were found within running times of some seconds, in contrast to several hours needed to find optimal solutions with the models.

\section{Illustrative numerical results}

This section evaluates the performance of ELISA implemented through each of the above SA schemes, considering a multi-hour traffic profile scenario when the spectral width of the slots was fixed to $12.5 \mathrm{GHz}$. Three national optical network topologies were used: the 21-node and 35-link Spanish Telefónica (TEL) topology, the 20-node and 32-link British Telecom (BT) topology, and the 21-node and 31-link Deutsche Telecom (DT) topology. Fig. 2 shows such topologies.

A demands mix belonging to three different traffic profiles (TPs) was used, where the bandwidth requested by each demand at each time period is randomly generated within the interval between the values $h^{\min }$ and $h^{\max }$ with a granularity of $10 \mathrm{~Gb} / \mathrm{s}$. Specifically, we define the following intervals in Gb/s: TP-1 [10, 100], TP-2 [40, 200], and TP-3 $[100,400]$. Note that, since the bandwidth of each demand at a given time period is not correlated with previous periods, high bandwidth fluctuations are expected between consecutive time periods. Moreover, high difference exists between $h^{\min }$ and $h^{\max }$ values (between 4 and 10 times higher). With these values, the on average bit rate per demand is in the order of $150 \mathrm{~Gb} / \mathrm{s}$. Finally, we considered an optical spectrum width of $600 \mathrm{GHz}$.

Fig. 3 shows the spectrum efficiency (used spectrum w.r.t. allocated spectrum) of the considered SA schemes as a function of the offered load in the TEL network. The efficiency of the Semi-Elastic and Elastic schemes is very similar, in the order to $95 \%$, as a consequence of both schemes allocate spectrum for the bandwidth requested in each time period and the narrow slot width chosen. However, the spectrum efficiency of the Fixed SA scheme is in the order of $60-75 \%$ since the size of the allocated spectrum is statically computed. Besides, the spectrum efficiency increases with the offered load.

Fig. 4 shows the percentage of un-served bandwidth (UBw) as a function of the total requested one for the three considered schemes. Considering $1 \%$ as the target un-served bandwidth for each of the networks, increments in the order of $17-20 \%$ of offered load can be obtained by applying the Semi-Elastic SA scheme with respect to the Fixed SA one. This is as a consequence of the elasticity that adding/releasing operations add to the former. Interestingly, the Elastic SA scheme adds equivalent increments (17-20\%) to the traffic server by the Semi-Elastic SA one, as a result of not only provide elastic spectrum allocation, but also dynamically changing the CF of the lightpaths.

In view of these results, and from the definition of the SA schemes where each scheme adds elasticity to the previous one, the following relation can be stated: $\mathrm{UBw}$ (Fixed SA) $\geq \mathrm{UBw}($ Semi-Elastic SA) $\geq \mathrm{UBw}$ (Elastic 

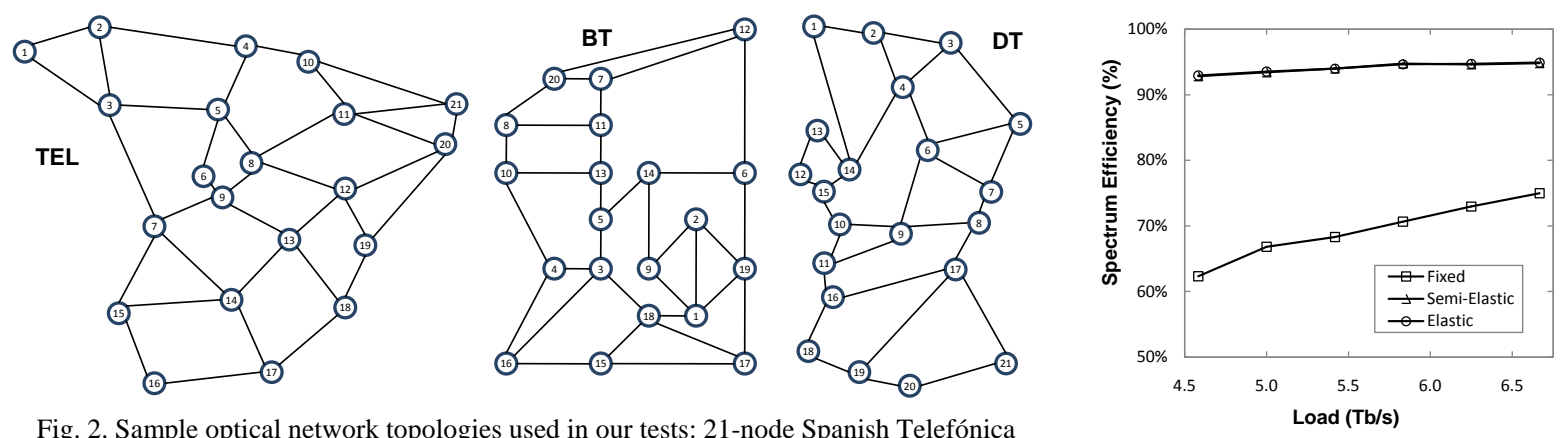

Fig. 2. Sample optical network topologies used in our tests: 21-node Spanish Telefónica (left), 20-node British Telecom (center), and 21-node Deutsche Telecom (right).
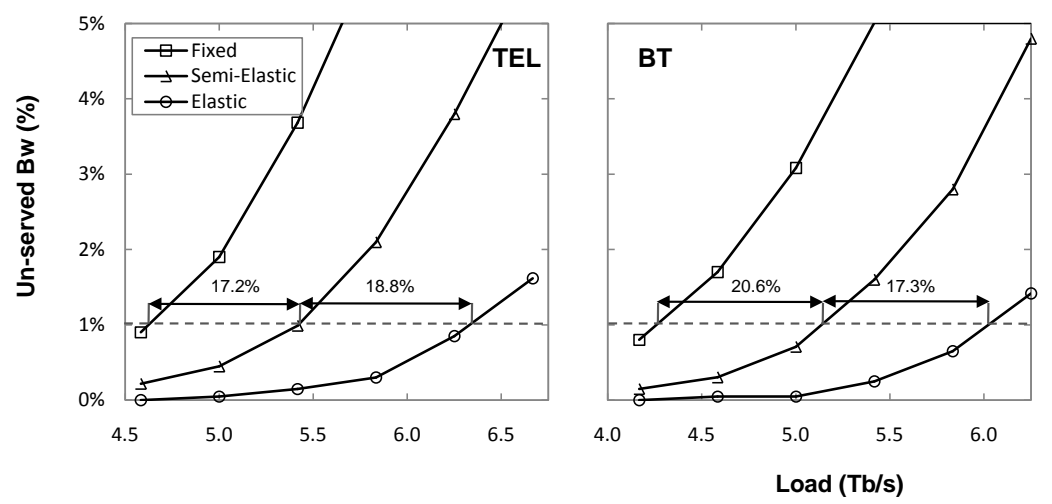

Fig. 3. Observed spectrum efficiency

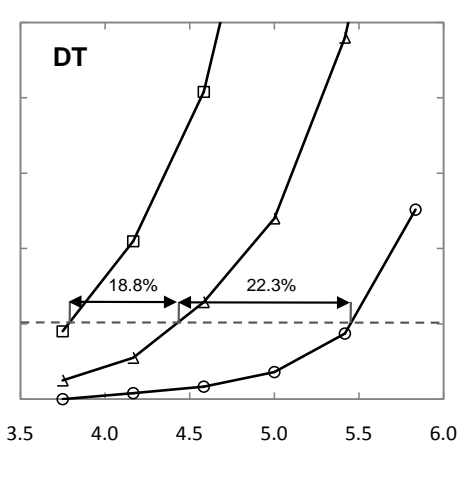

Fig. 4. Un-served bandwidth vs. offered load in TEL (left), BT (center), and DT (right) networks, for the three SA schemes considered. Relative gains are also highlighted.

SA). Note that in static scenarios where the requested bandwidth is constant with the time, that equality must be satisfied. Therefore, the gain of the Semi-Elastic and Elastic schemes is a function of the variability of the requested bandwidth in each time period.

\section{Conclusions}

In this paper we investigated the performance of three spectrum allocation schemes under variable in time traffic demands in EON. Obtained results show that the application of elastic spectrum allocation leads to a significant improvement in the network throughput with respect to fixed spectrum allocation when multi-hour traffic scenario are considered.

As a final remark regarding the above-defined SA schemes, the complexity of EON operation increases when the flexibility degree is increased. Table 1 briefly summarizes each scheme.

Table 1. Summary

\begin{tabular}{|c|c|c|}
\hline $\begin{array}{l}\text { Spectrum } \\
\text { Allocation }\end{array}$ & Requirements & Performance \\
\hline Fixed & No special requirements. & Low \\
\hline Semi-elastic & $\begin{array}{l}\text { - } \quad \text { Control plane protocols to allow dynamically modify the allocated spectrum. } \\
\text { - Optical transponders and filters to on-demand increase/decrease the used spectrum. }\end{array}$ & Medium \\
\hline Elastic & $\begin{array}{l}\text { - Control plane protocols to allow dynamically modifying the allocated spectrum and } \\
\text { the CF. } \\
\text { - Optical filters to on-demand increase/decrease the used spectrum. } \\
\text { - Optical transponders to on-demand modify the CF and/or increase/decrease the used } \\
\text { spectrum. } \\
\text { - Network management system to manage various demand changes in the network } \\
\text { simultaneously. }\end{array}$ & High \\
\hline
\end{tabular}

\section{References}

1. M. Jinno et al., "Spectrum-efficient and scalable elastic optical path network: Architecture, benefits, and enabling technologies," IEEE Comm. Mag., vol. 47, pp. 66-73, 2009.

2. M. Jinno et al., "Distance-adaptive spectrum resource allocation in spectrum-sliced elastic optical path network," IEEE Comm. Mag., vol. 48, pp. 138-145, 2010.

3. Y. Li, F. Zhang, and R. Casellas, "Flexible grid label format in wavelength switched optical network,” IETF RFC Draft, July 2011.

4. L. Velasco, M. Klinkowski, and M. Ruiz, "Modeling the Routing and Spectrum Allocation Problem for Future Elastic Optical Networks,” Technical Report UPC-DAC-RR-CBA-2011-8, 2011.

5. J. Gonçalves and M. Resende, “Biased random-key genetic algorithms for combinatorial optimization,” J. Heuristics, 2010. 